Tatiana A. Senina (moniale Kassia)

Saint Petersburg State University of Aerospace Instrumentation

Saint Petersburg, Russia

mon.kassia@gmail.com

\title{
DEUX LIVRES \\ SUR CASSIA DE CONSTANTINOPLE
}

Коста Симић, Иитургијска поэзија песникиње Касије и юен словенски превод, Нови Сад: Академскакњига, Београд: Институт за теолошкаистраживања, 2011, 248 с. ISBN 978-86-86611-88-8

[Kosta Simić, La poésie liturgique de la hymnographe Cassia et sa traduction slavonne, Novi Sad, Belgrade, 2011, 248 p.]

Ce livre très informatif est consacré à la poésie liturgique de $\mathrm{S}$. Cassia de Constantinople et prête une attention particulière à sa traduction slavonne. Il comporte une introduction, cinq chapitres, une conclusion, une bibliographie et un résumé en anglais.

Dans l'introduction l'auteur fait un bref exposé des études sur Cassia et son œuvre. On peut noter la lamentation bien justifiée au sujet des difficultés éprouvées par l'auteur lors de la recherche de la thèse de J. A. Bentzen "A Study of the Liturgical and secular Works of Blessed Kassia, Byzantine Nun and Poet $»{ }^{1}$ On peut remercier K. Simić pour un résumé de ce travail (p. 10-11).

Le chapitre I, « Живот песникиње Касије » (р. 14-19), représente un aperçu de la vie de la poétesse à la base des recherches précédentes et ne fournit aucun renseignement nouveau.

Dans le chapitre II, « Касијина дитургијска поезија » (р. 2030), l'auteur fait un court examen de la poésie liturgique de Cassia et de son usage à l'Église, en appelant aux témoignages des typika byzantins.

Ici et dans les chapitres suivants K. Simić n'accorde son attention qu'aux œuvres de Cassia qui passent sans aucun doute pour authen-

(1) University of New England, 1994. Quant à moi, je n'ai pas pu me procurer de ce travail. 
tiques:2 le canon des défunts, le tétraodion du Samedi Saint, les stichères en l'honneur des martyrs Gourias, Samonas et Habib (1 stichère), des martyrs Eustrate, Auxence, Eugène, Mardarios et Oreste (2), de la Nativité du Christ (9), de l'Épiphanie (3), de l'Hypapante (3), de la Nativité de Jean le Précurseur (1), des apôtres Pierre et Paul (1) et le fameux stichère du Mercredi Saint «Kú@ı $\alpha \mu \alpha \varrho \tau i ́ \alpha ı \varsigma ~ \pi \varepsilon \varrho ı \tau \varepsilon \sigma o \tilde{v} \sigma \alpha \gamma v v \eta ́$ ", en tout 2 canons et 21 stichères.

Le chapitre III, « Касијини канони » (р. 31-76), est consacré aux canons de Cassia. L'auteur nous donne des renseignements généraux sur le genre du canon (p. 31-35), le texte grec et la traduction serbe du canon des défunts avec une indication des citations et allusions bibliques (p. 35-48) et un commentaire, principalement théologique (p. 49-56). Le tétraodion du Samedi Saint est presenté d'une façon similaire : le texte grec et la traduction serbe (p. 56-60) et un beau commentaire (p. 60-76), y compris un aperçu de la doctrine orthodoxe sur la descente aux enfers et la résurrection du Christ, une étude du problème de la paternité des hyrmoi et l'indication des parallèles bibliques, hymnographiques et patristiques.

Le chapitre IV, « Касијине стихире » (р. 77-161), représente une étude des stichères précités de Cassia. L'auteur donne leur texte grec et traduction serbe avec des renseignements sur les fêtes ou saints correspondants, une analyse textuelle et un commentaire historique et théologique. Il indique les possibles parallélismes avec les Saintes Écritures, les œuvres des Pères, l'hymnographie (notamment la poésie de Romain le Mélode) et d'autres sources. Il semble pourtant que parfois K. Simić se soit trop intéressé à établir des parallèles : par exemple, la correspondance entre le stichère du Mercredi Saint et $l^{\prime}$ « Iliade » d'Homère n'est pas évidente (p. 84-85, n. 292); nous ne pouvons pas aussi être sûrs que Cassia connaissait réellement bien tous les hymnes de Romain le Mélode. Le commentaire sur le stichère de la Nativité de Jean le Précurseur (p. 138-154) et particulièrement intéressant : l'auteur y voit une espèce du credo ascétique de Cassia. Il est difficile de dater les œuvres de Cassia, mais la datation pour le stichère en l'honneur des apôtres Pierre et Paul de l'époque avant la fin du second iconoclasme (p. 161) semble juste.

(2) D'après l'étude d'Ilse RocHow, Studien zu der Person, den Werken und dem Nachleben der Dichterin Kassia (Berliner Byzantinische Arbeiten, 38), Berlin, 1967. 
Le chapitre V, «Сдовенски превод Касијине дитургијске поезије » (р. 162-211), est d'une grande valeur. Ici l'auteur examine les traductions des œuvres hymnographiques de Cassia en slavon d'après les manuscrits liturgiques des rédactions serbe et macédonienne. On n'y trouve que huit œuvres de la poétesse, dont une liste générale est à la page 164 du livre: le tétraodion du Samedi Saint, le stichère du Mercredi Saint et les stichères en l'honneur des martyrs Gourias, Samonas et Habib, des martyrs Eustrate, Auxence, Eugène, Mardarios et Oreste (deux stichères), de la Nativité du Christ

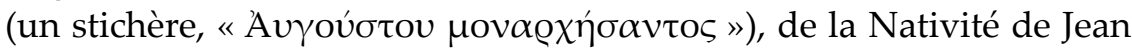
le Précurseur et des apôtres Pierre et Paul. L'auteur présente pour chaque œuvre le texte grec, le texte critique de la traduction slavonne et, pour celle-ci, un commentaire très détaillé sur le lexique, la syntaxe du cas et la syntaxe du verbe.

Dans la conclusion (p. 213-216) l'auteur fait des remarques finales sur Cassia comme hymnographe : elle était iconophile, connaissait bien l'Ancien et le Nouveau Testament, ainsi que l'héritage patristique, "lisait probablement les auteurs grecs classiques » et a subi l'influence des hymnographes byzantins de la première période; le trait distinctif de sa poésie est son caractère rhétorique et dramatique.

Le livre de Kosta Simić est un travail bien fait qui sera très utile à tous ceux qui s'intéressent à l'œuvre de Cassia et à l'hymnographie byzantine et sa réception slavonne en général.

\section{Kurt SHERry, Kassia the Nun in Context. The Religious Thought of a Ninth-Century Byzantine Monastic, Pis- cataway, NJ: Gorgias Press, 2011, xiv+146 p.}

\section{ISBN 978-1-61143-969-4}

Ce livre représente une tentative de résumer et systématiser la pensée de la fameuse femme-hymnographe Ste Cassia de Constantinople à la base de ses œuvres liturgiques et ses épigrammes gnomiques. Il comporte une introduction, cinq chapitres, deux appendices et une bibliographie. Dans la préface K. Sherry écrit : «I did not set out to undertake a comprehensive study of Kassia the Nun and her works. I was simply looking for a topic that would correlate my own research interests » (p. ix). En effet, ce livre n'est pas une étude fort approfondie, $c^{\prime}$ est plutôt une revue générale de la vie et de la pensée de Cassia dans le contexte de l'époque du second iconoclasme. 
Les détails de la biographie de Cassia sont examinés principalement dans l'introduction (p. 1-13) où l'auteur s'appuie sur les travaux d'I. Rochow et A. Silva et sur un Vie tardive de S. Cassia. K. Sherry pense que le nom monastique de Cassia était Cassienne, en l'honneur de S. Jean Cassien le Romain (p. 5), mais ce ne semble pas vraisemblable: dans le canon des défunts écrit par Cassia son nom

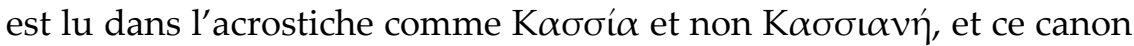
a été probablement écrit par la poétesse à un âge avancé, peut-être vers $860 . .^{3}$ Je noterais en passant que Sherry ne sait pas non plus que les théotokia de ce canon ne sont pas originales : il les examine comme des œuvres de Cassia (p. 57 et 59), tandis que la poétesse les a pris tous, à l'exception du théotokion de la 9ème ode, des canons différents qui se trouvent même jusqu'à présent dans les livres liturgiques. ${ }^{4}$

Le $1^{\text {er }}$ chapitre, "Kassia the Candidate" (p. 15-21), est consacré à la participation de Cassia à la cérémonie du choix de la fiancée par l'empereur Théophile. Pour une raison obscure Sherry date cet événement de 823 (p. 15, cf. p. 5). Cette date est donnée dans une version de la chronique de Syméon le Logothète comme l'année de l'avènement de Théophile au trône, mais c'est une erreur évidente ; Théophile est devenu co-empereur en 821 et empereur en $829,{ }^{5}$ et son mariage doit être daté de $821.6 \mathrm{~K}$. Sherry analyse le dialogue entre Théophile et Cassia d'une manière détaillée et comme un témoignage de l'éducation théologique de Cassia (p. 16-19), mais il ne connait pas les travaux de D. Afinogenov qui a montré que ce dialogue représente une citation d'une homélie sur l'Annonciation (BHG 1128f). ${ }^{7} \mathrm{Ce}$ dialogue témoigne vraiment de l'éducation de Cassia mais son sens

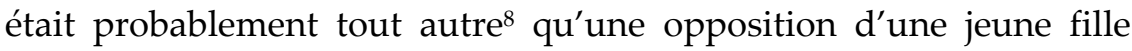

(3) T. A. Senina (moniale Kassia), « Remarques sur le canon des défunts écrit par S. Cassia de Constantinople», Scr, 5 (2009), pp. 319-322.

(4) Ibid., pp. 317-319.

(5) R.-J. Lilie, C. Ludwig, Th. Pratsch, I. ROCHOW, hg., Prosopographie der Mittelbyzantinischen Zeit. Erste Abteilung (641-867) (Berlin, 1998), \# 8167.

(6) Т. А. СенинА (монахиня Кассия), «Диалог Феофила и Кассии: литературная выдумка или реальность ? », Scr, 2 (2006), pp. 249-263.

(7) D. Afinogenov, «The Brid-Show of Theophilos: Some Notes on the Sourse », Eranos (Acta Philologica Suecana), 95 (1997), pp. 10-18; Д. АФИноГЕНОВ, Ю. КАЗАЧКОВ, «Дегенда о Феофиле: новые разоблачения», Ученые записки Российского Православного Университета ап. Иоанна Богослова, 5 (2000), c. 5-13.

(8) Voir : СенинА, « Диалог Феофила и Кассии », p. 263-270. 
orthodoxe et future «mère de l'Église » et de l'empereur "stupide », comme le pense Sherry (p. 19-21).

$\mathrm{K}$. Sherry note très justement que «the hymns of the Byzantine Church, especially those written between 500 and the fall of Constantinople to the Latins in 1204, offer a rich and much-neglected source for investigating Byzantine theology» (p. 10). À son avis, le contenu théologique de l'hymnographie de Cassia «place her, as a Church Mother, on par with those counted as Fathers » (p. 12). Sherry relève les sujets principaux des œuvres de Cassia - la femme dans le christianisme, l'iconophilie et la lutte contre l'hérésie, la vie monastique, l'amitié, la beauté et la santé, l'envie, la stupidité, le discours et le silence, - et cherche à donner un portrait de Cassia en tant qu'une personnalité.

Dans le $2^{\text {ème }}$ chapitre, «Kassia the Feminist » (p. 23-41), l'auteur examine les hymnes et les épigrammes de Cassia concernant les femmes et note que la poétesse n'est évidemment pas une féministe dans le sens moderne mais que "she reflects philosophically on the nature of gender and its function in Byzantine society » (p. 23). Selon Sherry, Cassia « is one of the few surviving female voices from the Byzantine era, and we find little evidence that she was a social rebel ", mais, comme la société byzantine était en général patriarcale, «Kassia clearly defied, or at least intellectually challenged, the norms of Byzantine society regarding women » (p. 23-24).

Certaines observations de l'auteur semblent intéressantes, mais tout de suite un grand défaut saute aux yeux: malheureusement, $\mathrm{K}$. Sherry ne s'est pas donné la peine d'étudier les travaux consacrés aux épigrammes de Cassia. Il continue de commenter son dialogue avec Théophile sans tenir compte de l'homélie comme sa source (p. 25, 27, 29), ne connait pas l'étude de M. Lauxtermann et E. Maltese sur Cassia $^{9}$ et, chose encore plus regrettable, il utilise la traduction

(9) M. D. LaUXTERMANN, The Byzantine Epigram in the Ninth and Tenth Centuries. A Generic Study of Epigrams and Some Other Forms of Poetry, Amsterdam, 1994, pp. 107-127 ; IDEM, Byzantine Poetry from Pisides to Geometres. Texts and Contexts, vol. I (Wiener byzantinistische Studien, XXIV/1), Wien, 2003, pp. 134, 241-270 ; E. V. MALTESE, « Una contemporanea di Fozio, Cassia. Osservazione sui versi profani », dans : La poesia tardoantica e medievale. Atti del I Convegno Internazionale di Studi. Macerata, 4-5 maggio 1998, ed. M. SALVADORE, Alessandria, 2001, pp. 71-83. 
assez inexacte des œuvres de Cassia faite par A. Tripolitis. ${ }^{10}$ Ainsi, Sherry écrit sur deux épigrammes «misogynes» de Cassia : «The verses suggest that an unattractive woman in Byzantium might justifiably lament the consequences of her ugliness, such as the reduction of her potential value in marriage, subjection to verbal abuse, and so on", dit Sherry. Il raisonne que pour Cassia "the fundamental problem with beauty is that it is "distracting" » : la beauté détourne l'homme de la contemplation de Dieu (p. 36). Mais pourquoi «distracting » ? Il s'agit des épigrammes suivantes :

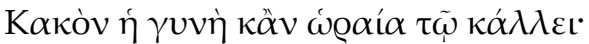

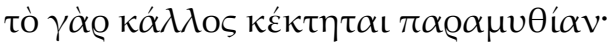

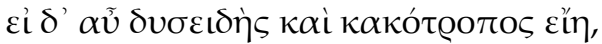

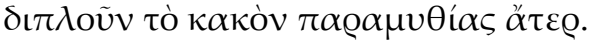

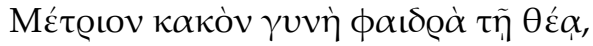

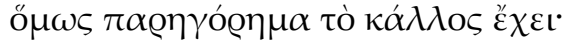

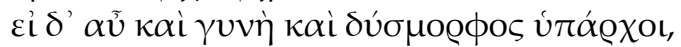

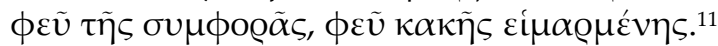

A. Tripolitis l'a traduis ainsi :

It is not good for a woman to be beautiful, for beauty is distracting; but if she is ugly and ill-mannered, without distraction it is twice as bad.

It is moderately bad for a woman to have a radiant countenance, Yet beauty has its consolation;

But if a woman is ugly,

What misfortune, what bad lack.12

Ni Sherry ni Tripolitis ne savent que ces épigrammes sont les paraphrases de deux passages du roman "Leucippe et Clitophon" d'Achille Tatius, comme l'a noté E. Maltese. ${ }^{13}$ En effet, on peut comparer :

(10) Dans le livre: A. TRIPOLITIS, Kassia: the Legend, the Woman, and her Work, New York, London, 1992.

(11) C 55-62 selon l'édition de K. KRUMBACHER, «Kasia », Sitzungsberichte der philosophisch-philologischen und der historischen Classe der k.b. Academie der Wissenschaften (1887), Heft. 1, S. 366-367.

(12) TRIPOLITIS, Kassia, p. 121.

(13) MALTESE, « Una contemporanea di Fozio, Cassia », p. 77-78. 


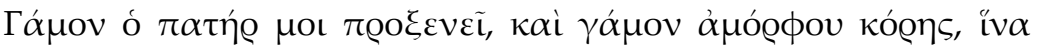

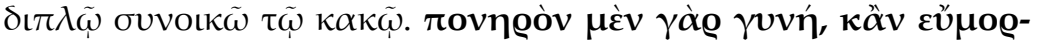

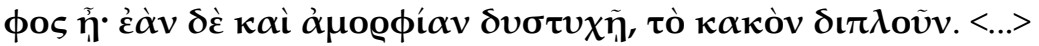

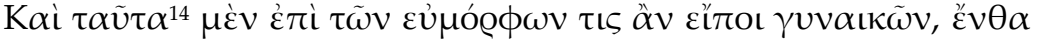

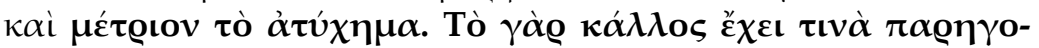

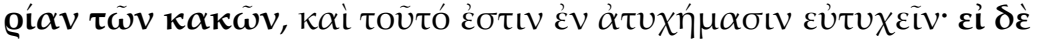

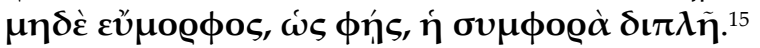

Il est évident qu'il s'agit ici d'une belle femme (et plutôt d'une épouse) comme consolation et non pas comme distraction. Achille Tatius emploie le mot $\pi \alpha \varrho \eta \gamma o \varrho i ́ \alpha$, Cassia le remplace par $\pi \alpha \varrho \alpha \mu v \theta i ́ \alpha$, mais le sens et le même. ${ }^{16}$ Ainsi, le contexte rend peu probable le raisonnement de K. Sherry sur "problem with beauty " pour Cassia "for beauty is distracting ». Je suis inclinée à admettre que ces deux épigrammes-paraphrases ont plutôt un caractère burlesque. ${ }^{17}$

Le même problème existe avec l'interprétation par Sherry de l'épigramme-paraphrase de 1 Esdras 3,12 :

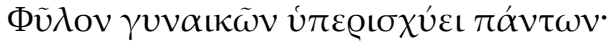

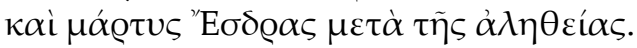

A. Tripolitis la traduit ainsi :

Esdras is witness that women

together with truth prevail over all. ${ }^{18}$

En appuyant sur cette traduction K. Sherry écrit : «Kassia composes her gnomic text to emphasize that women prevail when they act together with truth ». Ensuite il tire une parallèle entre cette épigramme

(14) Il s'agit des actions méchantes des femmes différentes.

(15) Achilles Tatius, Leucippe and Clitophon, ed. E. VILBORG, Stockholm, 1955, Book 1, Ch. 7.4.3-6 et 8.8.1-5 ; mis en gras par moi.

(16) П $\alpha \varrho \alpha \mu v \theta$ í $\alpha$ signifie comfort, consolation d'après G. W. H. LAMPE, A Patristic Greek Lexicon, Oxford, 1961, p. 1022, et comfort, benefit, help selon E. A. SOPHOCLES, Greek Lexicon of the Roman and Byzantine Periods (From B.C. 146 to A.D. 1100), Cambridge, Leipzig, 1914, p. 847.

(17) Elles suggèrent que Cassia ne trouvait pas le roman d'Achille Tatius trop « indécent », à la différence de Photius, mais l'a traité plus favorablement et avec humour, ce qui la rapproche à Léon le Mathématicien, comme j'ai déjà noté : T. A. SENINA (moniale Kassia), « Notices sur l'atmo-

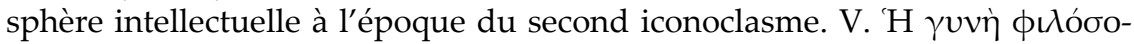
фos: S. Cassia de Constantinople et Platon », Scr, 4 (2008), pp. 339-340.

(18) TriPOLITIS, Kassia, p. 121. 
et un autre hymne écrit par Cassia ${ }^{19}$ et conclut : "Kassia's hymnography suggests that by embracing Truth (Christ) a women gains the strength required to overthrow the power of Satan » (p. 38).

Mais la traduction de l'épigramme est incorrecte : $\mu \varepsilon \tau \dot{\alpha} \tau \tilde{\eta} \varsigma \dot{\alpha} \lambda \eta$ $\theta \varepsilon i ́ \alpha \varsigma$ se rapporte à Ezras et non pas aux femmes. E. Maltese a remarquéz ${ }^{20}$ que $\alpha \lambda \eta \dot{\theta} \theta \varepsilon \iota \alpha$ ayant la valeur absolue chez Ezras se transforme en $\mu \varepsilon \tau \dot{\alpha} \tau \tilde{\eta} \varsigma \dot{\alpha} \lambda \eta \theta \varepsilon \dot{\alpha} \alpha \varsigma$ chez Cassia, ce qui suggère que la poétesse diminue l'emphase du texte biblique et, probablement, traite l'affir-

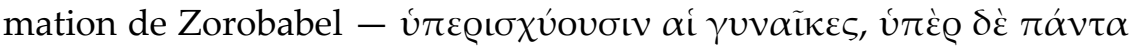

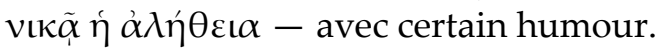

Il faudrait donc traduire l'épigramme ainsi :

The female race is the strongest one,

Ezra being the true witness of that.

Une autre singularité de l'étude de Sherry est qu'il cite partout les "poèmes » de Cassia : " in her poem "Woman" » (p. 35 et 38), «Kassia's poem "Friendship" » (p. 79), « in her poem "Man" » (p. 85), « her gnomic poem "Wealth/Poverty" »(p. 99) etc. Mais en réalité il n'existe pas de "poèmes » de Cassia. La poétesse écrivait de courtes épigrammes, et leurs collections dans les manuscrits diffèrent par leurs dimensions et leur composition. ${ }^{21}$ Ces épigrammes ne sont pas toujours classées par sujet dans les manuscrits. C'était A. Tripolitis qui les a classées ainsi, tout en détruisant l'ordre des épigrammes de l'édition ( $d$ 'après les manuscrits!) de K. Krumbacher. En effet, la collection "Woman », lignes 1-14, chez Tripolitis correspond aux vers C 43-46 et 53-62 d'après Krumbacher; la collection « Friendship », 1. 1-28, chez Tripolitis correspond aux vers A 1-26 et 31-32 selon Krumbacher ; la collection "Man » chez Tripolitis - aux vers A 93-106, 118-119 et 152-155 d'après Krumbacher; la collection «Wealth/Poverty» chez Tripolitis - aux vers A 56-63 et C 69-73 (c'est-à-dire de deux manuscrits différents !) d'après Krumbacher, etc. Il reste de poser la question si K. Sherry a jamais consulté l'édition de Krumbacher.

(19) En l'honneur de la grande martyre Barbe ; le hymne n'est attribué à Cassia que dans un seul manuscrit, ainsi sa paternité est incertaine, voir: I. Rochow, Studien zu der Person, den Werken und dem Nachleben der Dichterin Kassia, Berlin, 1967, S. 50, Nr. 33.

(20) MAlteSE, «Una contemporanea di Fozio, Cassia », p. 79.

(21) Voir : KRUMBACHER, «Kasia », S. 326-331 ; LAUXTERMANN, The Byzantine Epigram, pp. 110-113 ; IDEM, Byzantine Poetry, pp. 248-252. 
Dans le $3^{\text {ème }}$ chapitre, «Kassia the Iconophile» (p. 43-61), l'auteur examine la vie de Cassia pendant l'iconoclasme et sa participation à la résistance orthodoxe, en abordant également les rapports de Cassia avec ses parents qui n'étaient pas tous iconophiles. En se basant sur les œuvres de Cassia K. Sherry conclut qu'elle était iconophile et strictement orthodoxe, ce qui paraît juste. Sherry suppose que le stratège décédé mentionné dans une lettre de $S$. Théodore le Stoudite à Cassia'22 était le père de la poétesse (p. 44, n. 94, et p. 64). Cela me semble peu probable: cette hypothèse est dépourvue de fondement, et de plus, si le stratège avait été le père de Cassia, le ton de la lettre de Théodore aurait sûrement été moins sévère. Sherry écrit, en citant J. H. Rosser, sur les persécutions cruelles contre les iconophiles sous le règne de Théophile (p. 48). Mais l'ampleur de ces persécutions était exagérée par les sources iconophiles : en réalité cet empereur persécutait les iconophiles pour une raison plutôt politique que religieuse..$^{23}$ "One of the more infamous atrocities of Theophilos' Iconoclastic campaign ", dit Sherry, "was the branding of the Graptoi brothers, Theodore and Theophanes (later the famed chronicler known as Theophanes the Confessor) » (p. 48). Ici nous voyons peutêtre la plus grosse erreur dans le livre de Sherry : Theophane le Confesseur et Theophane le Graptos sont des personnes différentes! Le premier est l'auteur d'une chronique (terminée en 813 ou 814) et il était mort bien avant le règne de Théophile, en 818 ; le second n'a jamais écrit une chronique mais il est connu par ses œuvres hymnographiques.

Le $4^{\text {ème }}$ chapitre, «Kassia the Monastic » (p. 63-91), est consacré à la vie et à la philosophie monastique de Cassia. Il me semble le plus réussi dans le livre. K. Sherry examine les circonstances dans lesquelles Cassia a pris le voile, passe en revue les lettres de Théodore le Stoudite à Cassia (p. 63-68) et fait un aperçu du monachisme masculin et féminin à Byzance (p. 68-75). Ensuite l'auteur fait

(22) Ep. 539 d'après l'édition: Theodori Studitae Epistulae, hg. G. FATOUROS, Vol. II (Corpus Fontium Historiae Byzantinae, Series Berolinensis, 31.2), Berlin, New York, 1992, S. 813-814.

(23) Voir: J. GouILLARD, «La Vie d'Euthyme de Sardes († 831), une œuvre du patriarche Méthode », Travaux et Mémoires, 10 (1987), pp. 8, 14, 3840 ; А. Е. АФИНОГЕНОВ, «Повесть о прощении императора Феофила» $и$ Торжество Православия (Scrinium Philocalicum, IV), Москва, 2004, с. 51-53 ; T. A. SEninA (monial Kassia), « La confession de Théophane et Théodore les Graptoi : remarques et précisions », Scr, 4 (2008), pp. 286-290. 
une revue assez informative des épigrammes sur le moines et de quelques hymnes de Cassia en comparant leurs sujets avec la tradition patristique, avant tout avec l'Échelle sainte de Jean le Sinaïtique et les œuvres de Théodore le Stoudite (p. 75-91). Sherry met en évidence les thèmes principaux et les particularités de la philosophie monastique de Cassia et en vient à la conclusion suivante: "She drew heavily on the tradition she had received from the text of The Ladder of Divine Ascent and from her spiritual father, Theodore the Studite. However, she emphasized a contemplative, hesychastic monasticism rather than one of bodily praxis » (p. 90-91).

Dans le $5^{\text {ème }}$ chapitre, «Kassia the Person » (p. 93-110), l'auteur tâche d'éclaircir qui était Cassia en tant que personnalité. À la base de ses œuvres K. Sherry examine l'attitude de Cassia envers l'amitié, la beauté, la santé, les connaissances, la stupidité et l'envie, ses réflexions sur la loquacité et le silence. Sa conclusion sur la personnalité de Cassia est la suivante :

"While her strident tone in part reflects the didacticism of epigrams and Greek gnomic poetry in general, we definitely get the impression that Kassia, no matter how admirable her intelligence, was not an easy person to live with. She comes across as impatient, acerbic, and ... perhaps a bit self-righteous. At the same time, she appears to harbor a real desire for supportive bonds with others, advocating friendships in which both parties encourage and elevate one another spiritually. She often stresses the importance of harmonious relationships free of strife and bickering. ... Despite her obvious strength of character, she demonstrates little inclination to impose her views forcefully on others. ... Although one would hesitate to call her a moderate in her views, neither was she an extremist - she appears in her writings to be constantly seeking, if not a golden mean, at least an appropriate balance » (p. 109).

On peut probablement approuver dans l'ensemble de ce portrait, mais je voudrais noter que $l^{\prime}$ « intolérance » et $l^{\prime}$ " âpreté » vues dans les épigrammes de Cassia sont souvent caractéristiques des œuvres des auteurs byzantins en général, et il serait un peu injuste d'en accuser trop la poétesse.

Dans les appendices K. Sherry donne sa propre traduction anglaise des épigrammes de Cassia sur les moines, plus précise que celle d'A. Tripolitis (p. 117), et la traduction anglaise des passages des 
chroniques byzantines concernant Cassia (p. 119-132). Le livre se termine par une bibliographie (p. 133-145).

En résumant on peut dire que ce travail aurait pu devenir une étude généralisatrice importante sur Cassia et sa pensée, mais les erreurs fâcheuses et l'ignorance de quelques travaux importants précédents sur les œuvres de la poétesse a diminué considérablement la valeur du livre écrit par K. Sherry. 